\title{
A parametric LTR solution for discrete-time systems
}

\author{
Niemann, Hans Henrik; Jannerup, Ole Erik
}

Published in:

Proceedings of the 28th IEEE Conference on Decision and Control

Link to article, DOI:

10.1109/CDC. 1989.70160

Publication date:

1989

Document Version

Publisher's PDF, also known as Version of record

Link back to DTU Orbit

Citation (APA):

Niemann, H. H., \& Jannerup, O. E. (1989). A parametric LTR solution for discrete-time systems. In Proceedings of the 28th IEEE Conference on Decision and Control (Vol. Volume 1, pp. 481-482). IEEE.

https://doi.org/10.1109/CDC.1989.70160

\section{General rights}

Copyright and moral rights for the publications made accessible in the public portal are retained by the authors and/or other copyright owners and it is a condition of accessing publications that users recognise and abide by the legal requirements associated with these rights.

- Users may download and print one copy of any publication from the public portal for the purpose of private study or research.

- You may not further distribute the material or use it for any profit-making activity or commercial gain

- You may freely distribute the URL identifying the publication in the public portal

If you believe that this document breaches copyright please contact us providing details, and we will remove access to the work immediately and investigate your claim 


\title{
A PARAYEYRIC LTR-SOLUYIOY FOR DISCRETE-TIYE SYSTEYS.
}

\author{
Hans Henrik Niemann and ole Jannerup \\ Control Engineering Institute, Tech. University of Denmark \\ Technical University of Denmark \\ Building 424, DK-2800 Lyngby, Denmark.
}

ASTRACT.

A parametric LTR-solution for discrete-time compensator incorporating filtering observers which achive exact recovery will be presented for both minimum and non-minimum-phase systems.

First the recovery error. which define the difference between the target loop transfer and the full loop transfer function. Is manipulated into general form consisting of the tar get loop transfer matrix and the fundamental recovery matrix. Based on the recovery matrix a parametric LTR-solution will be developed. At last it will be shown that the LQG/LTR solution is included in this new parametric solution as spectal case.

1 Irrropuction.

The L,QG/LTR feedback design methodology for robust mode1based compensation for both continuous-time and discrete-time systems has received much attention in the recent years, see f.ex. $[1-10]$.

For continuous-time minimum-phase systems the asymptotic recovery techniques works very effective. Unfortunately similar asymptotic recovery technique is not feasible in discretetime. When the LQG/LTR-solution is applied to the discrete-time case, the recovery error will in general remains finite when a prediction observer is used, [6]. However, it is still possible to make LTR, but other methods must be applied. Based on the equation for the recovery error. a parametric exact LTR-solution has been developed in [9] for minimum-phase as well as non-minimum phase systems.

If the processing time of computing the control signal is negligible in comparison to the sampling interval, a filtering observer can be used in the compensator instead of a predition observer. It is then possible to achieve exact recovery in this case by using the LQG/LTR-solution when the system is minimum phase and has maximal number of zeros [7]. However, when these conditions isn't satisfied the LOG/LTR-solution wiIl result in . finite recovery error, but it will stili be possible to make exact recovery by applying the principple from the parametric LTR result in [ 9 ) to this case. It will then be possible to make $e-$ xact recovery for both non-minimum-phase systems as well as for systems with less than the maximal number of zeros.

\section{RBCOVERY BQUATION.}

In the following square discrete-time systems $s(A, B, C)$ are considered. The plant transter $\operatorname{mat} f(x) G(z)$ is given by:

$G(z)=C \Phi(z) B, \operatorname{dim} G(z)=m \times m$

$\Phi(z)=(\mathrm{Iz}-\mathrm{A})^{-1}, \mathrm{~d} \mathbf{m} \Phi(\mathrm{z})=\mathrm{n} \times \mathrm{n}$

It will be assumed that the model is minimal and let the number of transmission zeros be $p$. The plant is controlled by using a feedback compengator consisting of a filtering observer in serie with a state-feedback controller. [7] :

The compensator transfer matrix $H(z)$ can be rewritten as

$$
H(z)=2 K(I z-(I-F f C)(A-B K))-1 F^{f}
$$

where $F$ is the full-oxder observer gain, Ff is the filtering observer gain $($ the relation between $F$ and $F f$ is: $F=\lambda F f$, [7]) and $K$ in the full-state feedback gain.

Now let the two step LTR-design method $[1,2,3,6,7]$ be used for the design of $H(z)$ which consiat of target design of one of the compensator gains ( $K$ or $F$ ) followed by a recovery design of the other gain.

In order to formulate the loop-shape robustnesa constraints the uncertainties (disturbance, nolse and modelling errors) are reflected to the plant input [6]. The target loop transfer function 1 s then the full-state loop transfer $\mathrm{K} \$ \mathrm{~B}$ and the full $100 \mathrm{p}$ transfer is HG $[2,3]$. Let $\mathbb{E}_{I}(z)$ define the recovery error a. $[6,9]$ :

$E_{I}(z)=K \Phi(z) B-H(z) G(z)$

In order to have exact recovery it is required that $E_{I}(z)=$ 0 for all z. Now let eq. (3) be rewritten in an equpialent form:

$E_{I}(z)=M_{I}(z)\left\{I+M_{I}(z)\right]^{-1}[I+x \phi(z) \theta]$

where $M_{I}(z)$ is the recovery matrix defined by:

$M_{I}(z)=R^{f}(A-F C)(I z-A+F C)^{-1} B$

where $K=K^{f_{A}}$.

Proof of eq. (4) is given in the appendix.

Eq. (4) for the recovery error is equvialent to the equation defined in $[6,9]$ when prediction observer is used instead. The only difference is the equation for the recovery matrix.

It is simple to see by using the form of $B_{I}(z)$ in eq. (1) that:

$E_{I}(z)=0$ iff $M_{I}(z)=0$

The recovery matrix MI will only be used for making exact recovery in this paper, but $M_{I}$ have also other attractive properties. By using eq. (3) and (4) it is easy to derive that [10]:

$S_{I}(z)=S_{T F L}(z)\left(I+M_{I}(z)\right)$

where $S_{I}$ and $S_{T F L}$ are the input sensitivity functions for the full loop and the target full loop transfer function. For more details, please see $[10]$.

3 THE PARAMHTRIC LTR-SOLUTIOH.

Now let the equation for the recovery matrix, eq. $(5)$, be rewritten in the residual form, [6]:

$M_{I}(z)=K^{f} V W^{T}\left(I z-V n W^{T}\right)-1_{B}$

$$
=K_{i=1}^{n} \frac{v_{1} \tau_{1} v_{1} T_{B}}{z-\tau_{1}}
$$

where $v=\left[v_{1}, \ldots, v_{n}\right], w=\left[w_{1}, \ldots, v_{n}\right], \wedge=\operatorname{diag}\left(r_{1}, \ldots, \tau_{n}\right), v_{1}$ and $w_{1} T$ are right and left eigenvectors associated $w$ th the e1genvalue $\tau_{i}$ of $A-F C$. $V W^{T}=W^{T} V=I$

It is then easy to show that:

$M_{I}(z)=0$ iff

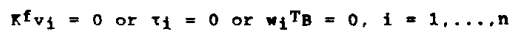

if A - FC is non-defective.

From eigenstructure assignment it is known that the left eigenvector $w_{i}{ }^{T}$ with the eigenvalue $\tau_{1}$ of $\mathrm{A}-\mathrm{FC}$ are given by [11]:

$$
\left[u_{1} T z_{1}^{T}\right]\left[\begin{array}{c}
\tau_{1} T-A \\
-C
\end{array}\right]=0,1=1, \ldots, n
$$


$\left[w_{10} z^{T} \quad z_{10}^{T}\right]\left[\begin{array}{cc}x_{10}-A & B \\ -C & 0\end{array}\right]=0$

Maximally $p$ eigenvectors $W_{i} 0^{T}$ can satisfy this condition, if $\tau_{10}$ is selected as transmission zero of $S(A, B, C)$, [14]. Let these $p$ eigenvalues/vectors be selected from eq. (11) so the $p$ conditions in eq. (9) are satisfied.

The second condition in eq. (9), $\tau_{i}=0$, can be satisfied by placing maximally $m$ eigenvalues $\tau_{1}$ at the origin.

Again from elgenstructure assignment [11] it is easily found that with $\tau_{1}=0$ :

$w_{1} T^{T}=z_{1}{ }^{T} C A^{-1}, \quad 1=p+1, \ldots, p+m$

The choice of $z_{i}^{T}$ depends on the type of the system which is treaded: A square uniform rank system or a square non-uniform rank system.

The non-uniform rank case.

The square non-uniform $x$ ank case is defined as:

$C_{A^{1}} B=0,1=1, \ldots, \alpha-2 \quad C_{A^{\alpha-1}} B \neq 0, \operatorname{det}\left[C^{\alpha-1} B\right]=0 \quad(13)$

The m eigenvectores ${ }_{1}{ }^{T}$ corresponding to the $m$ zero eigen values are given by, [13]:

$w_{10} O^{T}=e_{1-p^{T E C A}-1,} \quad i=p+1, \ldots p+m$

where $E$ is a $m x$ non-singular matrix of the appropriate coefficlents of the linear combination of the rows of $\mathrm{CA}^{-1}$. see shaked [13] for calculating $\mathrm{g}$.

Again $F$ can be parametrized by:

$$
F-\left[\begin{array}{c}
w_{1} T \\
\vdots \\
w_{n} T
\end{array}\right]^{-1}\left[\begin{array}{c}
z_{1} T \\
\vdots \\
z_{n} T
\end{array}\right]
$$

$z_{i}^{T}=z_{10} 0^{T}, \quad w_{i}^{T}=z_{10} T_{C}\left(\tau_{10} T-A\right)^{-1}, \quad i=1, \ldots p$

$z_{i}^{T}=e_{i}-p^{T} E, w_{i}^{T}=z_{i}{ }^{T} C_{A}-1$.

$1=p+1, \ldots, p+m$

and $\left(\tau_{i}, z_{i}{ }^{T}, 1=p+m+1, \ldots, n\right)$ are free design parameters.

The uniform_rank system.

The square uniform rank case is defined as, see [13]:

$C_{A} i_{B}=0,1=1, \ldots, \alpha-2, \operatorname{det}\left[C_{A}^{\alpha-1} B\right] \neq 0$

The solution for the uniform rank case is given by eq. (15) with $E=I$.

If $p<n-m$ the remaining $n-p-m$ conditions in eq. (9) must be satisfied by selecting $\mathrm{r}^{f}$ suitable. The first condition in eq. (9) implies:

$$
K^{f}\left[v_{1}, \ldots, v_{n}\right]=\left[\begin{array}{ll}
0 & 0
\end{array}\right]
$$

with $\operatorname{dim} Q=m \times(p+m)$ but otherwise it is arbitrary. Now

$$
\mathbf{x}=\left[\begin{array}{lll}
0 & 0
\end{array} \mathbf{V}^{-1} \mathbf{A}=0\left[\begin{array}{c}
w_{1} 0^{T} \\
\vdots \\
w_{p+m} 0^{T}
\end{array}\right] A=\operatorname{arA}\right.
$$

with dim $r=(p+m) \times m$. $r$ consist of the left eigenvectors $w_{10}$ constrained in eq. (11) and in eq. (14) and is thus a matrix of fixed elements.

A simple parametrization of the controller matrices which achleves exact recovery are found. An equvialent LTR result can be found in [9] when a prediction or a minimal-order observer are used.

1 Discussion

In this section few important consequences of exact LTR are discussed.

If the rank $[C B]=m$, i.e. $S(A, B, C)$ have maximal number of zeros, and $S(A, B, C)$ is minimum-phase, the $n$ recovery conditions in eq. ( 9 ) can be satisfy by the selection of $F$, and $K \mathrm{w} 111$ be free to select. A more aimple equation for $r$ than eq. (15) is given by: which is also the LQG/LTR solution [7], which show that the LQG/LTR solution 1s included as a special case.

If the LTR-results from sec. 3 are used on non-minimum phase system $G(z)$, the resulting controller will be unstable. It is, however, still possible to achieve exact recovery for nonminimum phase systems. In order to facilltate exact recovexy. note that in the selecting of $F$, only a subset $f$ of the eigenvectors constratined by eq. (11) need to be chosen. The consequence is, of cause, a reduction of the free parameters in $x$. Therefore such a selection is only advisible for non-minimum phase system if only the system's $f$ minimum phase zeros are used in eq. (11). By doing this the following equation will be satisfied by a stable compensator:

$K \Phi(z) B=H(z) G(z)$

The non-minimum phase zeros of $G(z)$ are not cancelled out on the right hand stde. Hence $H G$ and $\mathrm{K} \$ \mathrm{~B}$ are both non-minimum phase. This result is in agreement with the result in [15].

The selecting af $F$ is only constrained by eq. (15) and stabllity can always be achived for the observer. The parametrization of the state-feedback matrix $K$ imply that all the closed loop eigenvalues cannot be assigned freely, and no stability guarantees are available.

If a prediction observer is used, it is simple to develop dual result for the plant output loop breaking point $[6,9]$ which be due to the duality between the prediction observer and the state-feedback scheme. However, in spite of the missing duality when filtering observer is used, it is atill possible to develop dual result for the plant output loop breaking point This can easily be shown by applying the observer gain $F$ as the target design instead of $k$. The recovery error defined at the plant output loop breaking point is then given by:

$\left.E_{O}(z)=C(z)\right) F-G(z) H(z)$

gq. (21) can also be rewritten into a form equvialent to eq. (4).

5 REYERENCES.

[1] J.C. Doyle and G. Stein,1981, IEEE TAC, AC-26, pp. 4-16.

[2] M. Athans, 1986, Proc. ACC, Seattle,WA,pp. 1289-1286.

(3) G. Stein and $M$. Athans, 1987, IEEE TAC,AC-32,pp. 105-114.

[4] H, Kwarkernaak and R. Sivan, 1972, New York, wiley.

[5] A. Saberi and P. Sannuti,1988, Proc.AcC, Atlanta,pp. 803-8.

[6] G.C. Goodman,1984, M.Sc. thesis, rep.no, LIDS-TH-1392, Lab. of Inf. and Dec, Syat., MIT,MA, USA.

[7] J.M. Maciejorsk1,1985, TEEE TAC, AC-30, Pp. 602-605.

[8] P. Spgatrd-Andersen,1986, Ph.p thesis, Control Engineering Inst. Technical Untversity of Denmark.

[9] H.H. Niemann and P. Søgaard-Andersen, 1988, Proc. ACC, Atlanta, pp. 2483-2489.

[10] P. Spgaard-Andersen and H.H. Niemann, 1989, ProC. ACC, Pittsburgh, p. 922-929.

[11] A.N. Andry fr., et.a1.,1983, IEEE TAES, AES-19, pp.711-29.

[12] E.J. Davison and S.H, Wang.1975, IEEE TAC, AC-20,pp.516-18.

[13] U. Shaked, 1985, IEEE TAC, AC-30, pp. 34-47.

[14] U. Shaked and N. Karcnias, 1976, Int. J. Control,23,1, pp. 113-135.

[15] Z. Zhang and J.S. Freudenberg,1987, Proc. IEEE CDC, LOA Angeles, CA, Pp. $956-957$.

APPEMPIX.

Proof of eq. (4).

$\mathbf{B}_{I}(z)=\mathbb{R}\left(I-z\left(I z-\left(I-F^{f} C\right)(A-B K)\right)-F^{f} C\right](I z-A)-1_{B}$

$=R\left(I Z-\left(I-F^{f} C\right)(A-B K)\right)-1\left[I Z-\left(I-F^{f} C\right)(A-B K)-2 F^{f} C\right](I z-A)-1 B$

$=R(I z-(I-F f C)(A-B R))-1(I-F f C)(I Z-A+B R)(I z-A)-1_{B}$

$=R^{f}\left(I z-(A-F C)\left(I-B K^{f}\right)\right)^{-1}(A-F C)(B+B R(I z-A)-1 B)$

$=R^{f}(A-F C)\left(I Z-\left\{I-B K^{f}\right)(A-F C)\right)-1 B(I+K \Phi(z) B)$

$=R^{f}(A-F C)(I Z-A+F C)^{-1}\left(I+B K^{f}(A-F C)(I Z-A+F C)-1\right)-1 B(I+R \Phi B)$

$=K^{f}(A-F C)(I Z-A+F C)^{-1} B\left(I+R^{f}(A-F C)(I Z-A+F C)-1 B\right)-1(I+K \Phi B)$

$=M_{I}(z)\left(I+M_{I}(z)\right)-1(I+K \$ B)$ 